\title{
Self image, stress level and quality of life in adolescents patients with idiopathic scoliosis
}

\author{
E Kinel $^{1 *}$, T Kotwicki ${ }^{2}$, A Podolska ${ }^{1}$, M Białek ${ }^{3}$, W Stryła ${ }^{1}$ \\ From 9th International Conference on Conservative Management of Spinal Deformities - SOSORT 2012 \\ Annual Meeting \\ Milan, Italy. 10-12 May 2012
}

\section{Background}

Adolescent idiopathic scoliosis (AIS) is a complex and progressive condition, which can affect a patient's quality of life (QoL).

\section{Aim}

The aim of the study was to evaluate the self-image, the stress level, and the quality of life in adolescents with idiopathic scoliosis, who are under brace treatment.

\section{Methods}

It involved 59 adolescents, (50 girls, 9 boys), ages ranging between 10.0 and 17.0 years, all with Adolescent Idiopathic Scoliosis (AIS) with Cobb angle between 20-58 degrees. The adolescents were wearing the same kind of brace (Chêneau orthosis) for more than 6 months, for at least $16 \mathrm{~h}$ per day. Three questionnaires were used: Trunk Appearance Perception Scale (TAPS), Bad Sobernheim Stress Questionnaire (BSSQ Brace, BSSQ Deformity), and Brace Questionnaire (BrQ).The TAPS is a tool to evaluate subjective impression of trunk deformity, which includes 3 sets of figures that depict the trunk, from 3 viewpoints: looking toward the back, looking toward the head with the patient bending over, and looking toward the front. BSSQ is the questionnaire that estimates the stress scoliosis patients have, whilst wearing their brace. The BrQ is an instrument for measuring quality of life of scoliotic adolescents, who are being treated conservatively, with wearing of a corrective brace. The analysis considered the type of treatment, curve location, correlation of the total scores with age, Cobb angle and Bunnell rotation angle.

\section{Results}

The age was $14.1 \pm 1.7$ years. Cobb angle was $33.2 \pm 9.5$ degrees. The TAPS median for the total score was 4 . Adolescents revealed higher score with BSSQ Deformity $($ median $=14)$ comparing to BSSQ Brace $($ median $=11)$. The mean score for BrQ was $76.6 \pm 12.1$.

\section{Conclusion}

The adolescents who were under brace treatment suffered moderate level of stress from the deformity. Conservative treatment did not severely impact on the quality of life of scoliotic adolescents.

\section{Author details}

${ }^{1}$ Department of Rehabilitation, University of Medical Sciences, Poznan, Poland. ${ }^{2}$ Department of Paediatric Orthopaedics and Traumatology, University of Medical Sciences, Poznan, Poland. ${ }^{3}$ FITS Center, Jawor, Poland.

Published: 3 June 2013

\section{References}

1. Bago J, Sanchez-Raya J, Perez-Grueso FJ, Climent JM: The Trunk Appearance Perception Scale (TAPS): a new tool to evaluate subjective impression of trunk deformity in patients with idiopathic scoliosis. Scoliosis 2010, 5:6.

2. Vasiliadis E, Grivas TB, Gkoltsiou K: Development and preliminary validation of Brace Questionnaire (BrQ): a new instrument for measuring quality of life of brace treated scoliotics. Scoliosis 2006, 1:7.

3. Botens-Helmus C, Klein R, Stephan C: The reliability of the Bad Sobernheim Stress Questionnaire (BSSQbrace) in adolescents with scoliosis during brace treatment. Scoliosis 2006, 1:22.

doi:10.1186/1748-7161-8-S1-057

Cite this article as: Kinel et al:: Self image, stress level and quality of life in adolescents patients with idiopathic scoliosis. Scoliosis 2013 8(Suppl 1): O57.

'Department of Rehabilitation, University of Medical Sciences, Poznan,

Poland

Full list of author information is available at the end of the article

(c) 2013 Kinel et al; licensee BioMed Central Ltd. This is an Open Access article distributed under the terms of the Creative Commons 